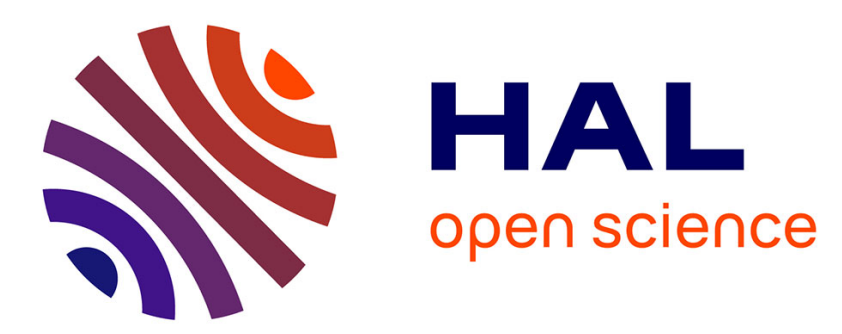

\title{
Propriétés de luminescence des ions de terres rares dopés dans des nanoparticules diélectriques incorporées dans une matrice vitreuse
}

Wilfried Blanc

\section{- To cite this version:}

Wilfried Blanc. Propriétés de luminescence des ions de terres rares dopés dans des nanoparticules diélectriques incorporées dans une matrice vitreuse. Matériaux \& Techniques, 2015, 103 (4), pp.4074016. 10.1051/mattech/2015049 . hal-01227680

\section{HAL Id: hal-01227680 \\ https://hal.science/hal-01227680}

Submitted on 11 Nov 2015

HAL is a multi-disciplinary open access archive for the deposit and dissemination of scientific research documents, whether they are published or not. The documents may come from teaching and research institutions in France or abroad, or from public or private research centers.
L'archive ouverte pluridisciplinaire HAL, est destinée au dépôt et à la diffusion de documents scientifiques de niveau recherche, publiés ou non, émanant des établissements d'enseignement et de recherche français ou étrangers, des laboratoires publics ou privés. 


\title{
Propriétés de luminescence des ions de terres rares dopés dans des nanoparticules diélectriques incorporées dans une matrice vitreuse.
}

\author{
Wilfried Blanc \\ Université Nice Sophia Antipolis, CNRS, LPMC, UMR 7336, 06100 Nice, France
}

résumé (150-200 mots) : Les ions de TR sont des éléments chimiques uniques couvrant un très large éventail d'applications. En particulier, ils ont permis de nombreuses applications innovantes dans le domaine de la photonique. Afin de développer de nouvelles fonctionnalités, un intérêt croissant réside dans l'utilisation de nanoparticules dopées d'ions de terres rares, qu'il s'agisse des nanoparticules utilisées pour l'imagerie médicale ou des nanoparticules contenues dans un matériau comme dans les vitro-céramiques. Cet article propose de faire le point sur les propriétés spectroscopiques des ions de terres rares dans ces différents milieux. En particulier, nous discuterons des modifications induites lors de l'insertion des ions de terres rares dans une nanoparticules puis lors de l'insertion de ces mêmes nanoparticules dans une matrice de verre. La compréhension de ces différents phénomènes ouvre de nouvelles perspectives pour la photonique.

mots-clés : ions de terres rares, luminescence, nanoparticules, vitro-céramiques

\section{Introduction}

Découverts à la fin du 18e siècle par C.A. Arrhenius, les ions de terres rares (TR) sont classés par l'Union internationale de chimie pure et appliquée (UICPA) comme comprenant les quinze lanthanides du tableau périodique (du lanthane au lutécium, les numéros atomiques $Z$ variant de 57 à 71), plus le scandium $(Z=21)$ et l'yttrium $(Z=39)$. Ces métaux sont abondants dans la croûte terrestre, chacun en quantités variables; leur abondance individuelle diminue à mesure que leur nombre atomique augmente [1].

Les ions de TR sont des éléments chimiques uniques, couvrant un très large éventail d'applications. Ils sont utilisés pour la fabrication d'aimants permanents (principalement Nd, Pr et Dy) et sont à la base du développement d'applications révolutionnaires tels que les véhicules électriques et les éoliennes. Les ions de TR sont également employés comme catalyseurs dans l'industrie pétrolifère. À l'instar de $\mathrm{CeO}_{2}$, ce sont des agents de polissage couramment utilisés pour le verre. Dans cet article, nous nous intéressons plus particulièrement à leurs propriétés de luminescence qui ont permis de nombreuses applications innovantes dans le domaine de la photonique [2,3]. Par exemple, les luminophores convertissent la lumière UV en lumière visible grâce aux ions $\mathrm{Eu}^{2+}$ (bleu), $\mathrm{Tb}^{3+}$ (vert) et $\mathrm{Eu}^{3+}$ (rouge). Les ions de $\mathrm{TR}$ sont particulièrement utilisés dans des amplificateurs optiques et des lasers, comme par exemple dans le YAG: $\mathrm{Nd}^{3+}$ qui émet à $1064 \mathrm{~nm}$. Ils trouvent aussi des applications dans des verres comme dans le cas des des fibres optiques amplificatrices à base de silice ou les lasers de puissance $\left(\mathrm{Er}^{3+}, \mathrm{Yb}^{3+}, \mathrm{Tm}^{3+}\right)[4]$.

Comme nous le verrons dans la suite de l'article, les propriétés de luminescence dépendent très fortement du matériau dans lequel ils sont insérés. Le choix du matériau hôte résulte toujours d'un compromis entre l'impact économique, la fiabilité, l'aptitude à être mis en forme dans des formes 
désirées et divers autres facteurs. De ce point de vue, l'incorporation de nanoparticules dopées d'ions de TR dans un matériau vitreux offre de nombreux avantages. En effet, les vitro-céramiques peuvent être facilement mises en forme et sont plus faciles et moins coûteuses à produire que les matériaux monocristallins. Elles présentent également une bonne durabilité chimique et potentiellement une bonne transparence. Ainsi, la propriété optique du matériau résultant provient des ions de TR présents dans les nanoparticules incorporées dans le matériau vitreux; la composition chimique des nanoparticules est choisie spécifiquement en fonction des propriétés de luminescence recherchées. La première vitro-céramique avec des propriétés optiques a été proposée dès 1975 [5]. Il s'agissait de verres d'oxydes contenant des particules fluorées $\left(\mathrm{PbF}_{2}\right)$ dopées avec des ions de terres rares $\left(\mathrm{Er}^{3+}, \mathrm{Yb}^{3+}\right)$. La taille microscopique des cristaux induisait une forte diffusion de la lumière. C'est en 1993 que la première vitro-céramique transparente est obtenue (matrice silicatée contenant des nanoparticules fluorées dopées $\mathrm{Er}^{3+}-\mathrm{Yb}^{3+}$ ) [6]. Depuis, les vitrocéramiques oxyfluorées ont fait l'objet de nombreuses études ainsi que des nanoparticules d'oxydes dans un verre d'oxyde (comme par exemple la formation de nanoparticules de forsterite $\left(\mathrm{Mg}_{2} \mathrm{SiO}_{4}\right)$ dans un silicate) $[7,8,9,10]$. Le choix d'une nanoparticule porte généralement sur sa composition par rapport aux propriétés optiques recherchées, la taille visée étant a priori la plus petite possible afin de limiter les pertes par diffusion de lumière. Nous verrons dans cet article que pour les petites nanoparticules, il convient aussi de considérer la matrice hôte car elle influence les propriétés optiques des ions de terres rares bien que ceux-ci soient localisés dans les nanoparticules.

Les propriétés de luminescence des ions de TR insérés dans un matériau massif sont rappelées dans le paragraphe 2. Les modifications apportées à ces propriétés, lorsque le matériau massif est réduit à l'échelle du nanomètre (les effets de confinement) sont examinées dans le paragraphe 3 . Enfin, le paragraphe 4 se concentre sur les propriétés optiques des nanoparticules dopées d'ions de TR lorsqu'elles sont incorporées dans une matrice de verre.

\section{Propriétés optiques des ions de terres rares dans un matériau massif}

Les propriétés optiques des ions de TR découlent de leur configuration électronique [2,4]. La configuration électronique des ions de TR est $[\mathrm{Xe}] 4 f^{1} 5 d^{1} 6 s^{2}$, où $[\mathrm{Xe}]$ correspond à la configuration électronique du xénon et $n$ le nombre d'électrons de la couche électronique $4 f, n$ variant de 0 (pour le lanthane) à 14 (pour le lutécium). Lorsque les ions sont insérés dans un verre ou un cristal, l'état d'oxydation prépondérant est trivalent et les couches électroniques $5 d$ et $6 s$ sont vides. Les états bivalent $\left(\mathrm{Eu}^{2+}, \mathrm{Yb}^{2+}\right.$ et $\left.\mathrm{Sm}^{2+}\right)$ et tétravalent $\left(\mathrm{Ce}^{4+}, \mathrm{Pr}^{4+}\right.$ et $\left.\mathrm{Tb}^{4+}\right)$ sont aussi observés pour quelques ions [11].

Les propriétés de luminescence des ions de TR sont issues des transitions électroniques intraconfigurationelles $\left(4 f^{n} \rightarrow 4 f^{n}\right)$ et interconfigurationelles $\left(4 f^{n-1} 5 d^{1} \rightarrow 4 f^{n}\right)$. Dans ce dernier cas, l'émission est dans le domaine bleu-UV (pour les ions $\mathrm{Ce}^{3+}, \mathrm{Pr}^{3+}$ ou $\mathrm{Eu}^{2+}$ ) ou à plus haute énergie (pour tous les autres ions de TR) alors que les transitions $4 f-4 f$ couvrent la gamme visible - IR. Dans cet article, nous nous intéresserons plus particulièrement aux transitions $4 f-4 f$.

\subsection{Structure des niveaux d'énergie}

Afin de déterminer la structure des niveaux d'énergie des ions de TR, le hamiltonien est décrit 
comme suit:

$$
H=H_{0}+H_{e l}+H_{S O}+H_{C F}
$$

où $H_{0}+H_{e l}+H_{S O}$ est le hamiltonien de l'ion libre $\left(H_{0}\right.$ est le hamiltonien de l'ion libre, $H_{e l}$ représente la répulsion électrostatique entre les électrons, $H_{S O}$ est le terme de couplage spin-orbite) et $H_{C F}$ représente l'interaction entre les ions de TR et les ions environnants de la matrice hôte [2,4]. La couche électronique $4 f$ est à l'intérieur des couches pleines $5 s^{2} 5 p^{6}$. Compte tenu de cet écrantage, les électrons de la couche $4 f$ ont une interaction faible avec les atomes de la matrice hôte $\left(H_{S O}>H_{C F}\right.$, approximation du champ faible). Le diagramme des niveaux d'énergie est représenté Fig. 1.

L'influence de l'hôte est faible, conduisant les multiplets ${ }^{2 \mathrm{~S}+1} \mathrm{~L}_{\mathrm{J}}$ à se scinder en sous-niveaux Stark, généralement séparés par 10-100 $\mathrm{cm}^{-1}$ (pour comparaison, les états SO sont séparés par 1000-10000 $\left.\mathrm{cm}^{-1}\right)$. La dégénérescence dépend fortement de la symétrie du site occupé par l'ion de TR. La levée de dégénérescence est quasi-complète dans le cas des verres en général (faible symétrie du site). En changeant le matériau, la position des niveaux Stark est modifiée et par conséquent l'énergie des transitions. La position des états SO est également impactée par la nature covalente de l'interaction TR-hôte (par exemple, la distribution partielle de la densité électronique de l'ion de TR sur les atomes environnants, semblable à celle des atomes d'oxygène dans les matériaux d'oxyde). Cependant, cet effet, connu comme l'effet néphélauxétique, est plus prononcé pour les électrons $5 d$ parce qu'ils ne sont pas écrantés par les couches pleines $5 s^{2} 5 p^{6}$, contrairement à ce qui se passe pour les électrons de la couche $4 f[12]$. A titre d'exemple, la transition $4 f-5 d \mathrm{de}^{3 \mathrm{Ce}^{3+}}$ donne lieue à une émission UV dans les fluorures et rouge dans les sulfures. Dans le cas des transitions $4 f-4 f$, la longueur d'onde centrale de la bande d'émission est très peu modifiée en changent le matériau hôte.

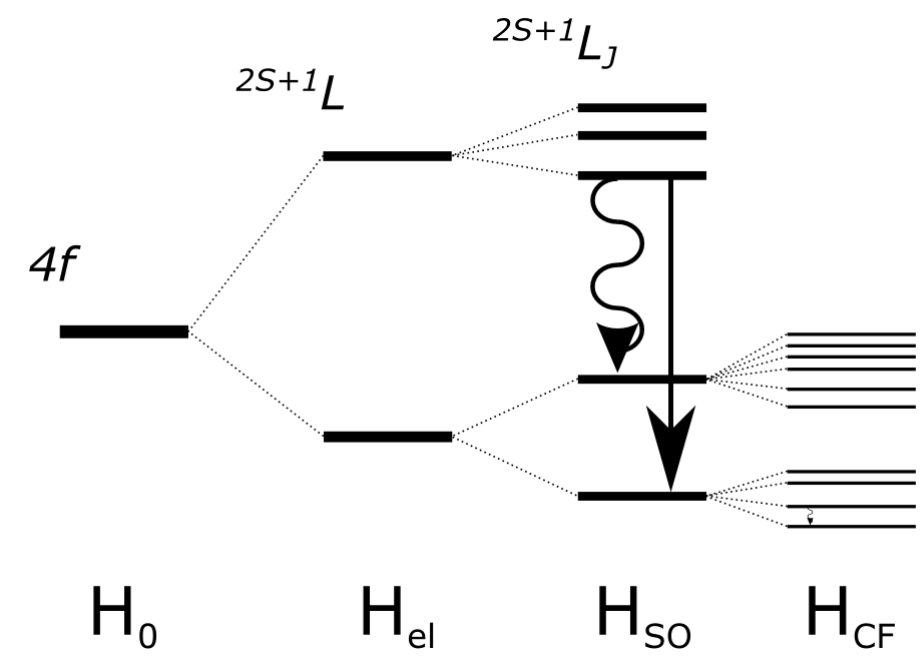

Fig. 1. Diagramme des niveaux d'énergie d'un ion de TR illustrant l'éclatement des niveaux induit par les différentes interactions (cf texte). La flèche droite représente une transition radiative entre des niveaux ${ }^{2 S+1} \mathrm{~L}_{\mathrm{J}}$ et la flèche ondulée correspond à une transition non-radiative entre des niveaux ${ }^{2 \mathrm{~S}+1} \mathrm{~L}_{\mathrm{J}}$ ou des sous-niveaux Stark. 


\subsection{Intensité des transitions}

Les transitions entre les niveaux électroniques sont de nature dipolaire électrique et magnétique. Hormis pour quelques transitions spécifiques telle que ${ }^{4} \mathrm{I}_{13 / 2} \rightarrow{ }^{4} \mathrm{I}_{15 / 2}$ pour $\mathrm{Er}^{3+}$ (d'intérêt pour les télécommunications), les transitions dipolaires magnétiques sont de plusieurs ordres de grandeur plus faibles que les transitions dipolaires électriques et ne seront pas traitées dans cet article [13].

La force $S_{a, b}$ d'une transition entre les états $a$ et $b$ est donnée par :

$$
S_{a, b}=\sum_{i, j}\left|\left\langle b_{j}|D| a_{i}\right\rangle^{2}\right|
$$

où $D$ est l'opérateur d'interaction dipolaire électrique et $i, j$ sont les sous-niveaux des états initiaux et finaux.

Dans le cas de transitions entre états $4 f$, les transitions dipolaires électriques sont interdites en raison des règles de sélection (pas de transitions entre états de même parité). Cependant, ces transitions sont observées parce que le champ cristallin mêle les états $5 d$ avec les états $4 f$. En d'autres termes, l'intensité de la transition est influencée par la capacité de l'hôte à mélanger les états de parité opposée. La force d'oscillateur $f_{a, \mathrm{~b}}$ est un paramètre sans dimension utilisé pour calculer l'intensité de transition radiative entre les états $a$ et $b$ et est exprimée comme:

$$
f_{a, b}=\frac{8 \pi^{2} m v}{h e^{2}} \frac{\chi_{L}}{n} \frac{1}{g_{a}} S_{a, b}
$$

où $h$ est la constante de Planck, $n$ est l'indice de réfraction de l'hôte, $v$ est la fréquence moyenne du photon, $g_{a}$ est la dégénérescence de l'état initial $a\left(g_{\mathrm{a}}=2 J_{\mathrm{a}}+1\right), m$ et $e$ sont la masse et la charge de l'électron et $\chi_{\mathrm{L}}$ est la correction de champ local. La correction de champ local se produit en raison de la différence de polarisabilité entre l'hôte et l'ion. Pour les transitions dipolaires électriques, la correction de champ électrique est la suivante :

$$
\chi_{L}=\left(\frac{n^{2}+2}{3}\right)^{2}
$$

Pour les ions de terres rares, les forces d'oscillateur sont typiquement de l'ordre de $\sim 10^{-6}$ (elles sont de $\sim 1$ pour les transitions autorisées).

\subsection{Durées de vie radiatives et non-radiatives}

L'interaction des ions de TR avec leur environnement impacte aussi la dynamique des états électroniques.

La luminescence comprend, par définition, à la fois la fluorescence et la phosphorescence. Ces mécanismes d'émission de lumière diffèrent par leur durée de vie. La fluorescence a une durée de vie d'émission courte (ns ou ps), alors que pour la phosphorescence la durée de vie d'émission est plus longue $(>\mathrm{ms})$. La durée de vie de fluorescence $(\tau)$ d'un niveau dépend à la fois de la durée de vie radiative $\left(\tau_{r}\right)$ et non-radiative $\left(\tau_{n r}\right)$ définie comme suit : 


$$
\frac{1}{\tau}=\frac{1}{\tau_{r}}+\frac{1}{\tau_{n r}}
$$

Plus précisément, la durée de vie radiative $\tau_{r}$ est définie par :

$$
\frac{1}{\tau_{r}}=A_{a, b}
$$

où le coefficient d'Einstein $A_{a, b}$ représente le taux de transition spontanée du niveau d'énergie $a$ à un niveau d'énergie $b$. Il est lié à la force d'oscillateur $f_{a, b}$ comme suit :

$$
f_{a, b}=4 \pi \epsilon_{0} \frac{m c^{2}}{8 \pi^{2} n e^{2} v^{2}} \frac{1}{\chi_{L}} A_{a, b}
$$

L'interaction de l'ion de TR avec les états de vibration de la matrice hôte est responsable de la durée de vie non radiative $\tau_{n r}$, avec une valeur de l'ordre de la picoseconde. Cette relaxation rapide se produit entre les sous-niveaux Stark où les différences d'énergie sont faibles. La population des niveaux Stark est donc principalement gouvernée par une statistique de Boltzmann, avec un taux de transition non-radiative $W_{n r}$ exprimé ainsi :

$$
\frac{1}{\tau_{r}}=W_{n r}=W_{0} \exp \left(-\alpha\left(\Delta E-2 E_{p}\right)\right)
$$

où $W_{0}$ et $\alpha$ sont des constantes caractéristiques de la matière hôte, $\Delta E$ est la différence d'énergie entre les deux niveaux pour laquelle la transition se produit et $E_{p}$ est l'énergie de phonon la plus élevée. L'énergie des phonons dépend de la matière hôte, et sa contribution influence grandement la nature radiative / non-radiative de la transition. Un exemple est le cas du niveau ${ }^{3} \mathrm{H}_{4}$ pour l'ion $\mathrm{Tm}^{3+}$. $4000 \mathrm{~cm}^{-1}$ sépare le niveau ${ }^{3} \mathrm{H}_{4}$ du niveau le plus proche d'énergie inférieure $\left({ }^{3} \mathrm{H}_{5}\right)$. Lorsque l'ion $\mathrm{Tm}^{3+}$ est incorporé dans la silice (l'énergie de phonon $E_{p}$ est de $1100 \mathrm{~cm}^{-1}$ ), la transition à partir du niveau ${ }^{3} \mathrm{H}_{4}$ est à $2 \%$ radiative tandis que dans les fluorures $\left(E_{p}=550 \mathrm{~cm}^{-1}\right)$, la transition est $\sim 100 \%$ radiative.

\section{4 Élargissements homogène et inhomogène des transitions}

Les élargissements homogène et inhomogène des niveaux d'énergie influent sur la forme de la bande d'émission et d'absorption.

L'élargissement homogène de la courbe d'émission est décrit par une fonction de Lorentz. Il s'explique par la durée de vie finie de l'état $(\Delta E . \Delta t \geq \hbar$, principe d'incertitude d'Heisenberg) et par l'interaction entre les ions de TR et leur environnement. L'interaction ion-phonon est un mécanisme dominant, dépendant de la température $T$, qui contribue à l'élargissement. Pour $T>>T_{\text {Debye }}(10-20$ $\mathrm{K}$ ), le processus Raman (mécanisme à deux phonons: un absorbé et un émis) est prédominant et la largeur de raie homogène varie en $T^{2}$. A $T<10 \mathrm{~K}$, la dépendance suit une loi de puissance en $T^{\alpha}$ avec $\alpha=1-2$ dans des verres [14]. Ce comportement est différent de celui d'un cristal et est expliqué dans le cadre du modèle à deux niveaux (discuté dans la section 4.2). La largeur de raie homogène est typiquement dix fois plus grande dans les verres que dans les cristaux.

L'élargissement inhomogène est causé par la variation du champ cristallin d'un site à un autre. Cette 
variation modifie l'éclatement Stark (voir Fig. 1) et aussi la force de la transition optique. Les transitions globales, issues des mêmes sous-niveaux Stark initiaux et finaux, sont bien décrits par une fonction gaussienne. En contraste avec l'élargissement homogène, l'élargissement inhomogène ne dépend pas de la température. Dans un cristal, les ions de TR sont dans un nombre restreint de sites cristallographiques et l'élargissement inhomogène est très faible, à la différence d'un verre pour lequel il y a une multiplicité de sites possibles. Ces différences se traduisent par des formes de spectres d'émission et d'absorption très différents : spectres de raies dans le cas des cristaux, spectres de bandes pour les verres.

Dans les verres, les élargissements homogènes et inhomogènes ont des valeurs comparables (environ de 10 à $100 \mathrm{~cm}^{-1}$ ) à température ambiante. La forme des transitions peut alors être décrite par une fonction de Voigt qui est la convolution d'une fonction gaussienne et lorentzienne.

\subsection{Transferts d'énergie}

Les sections précédentes ont décrit un ion de TR interagissant avec la matrice hôte. Des interactions TR-TR au sein de la matrice hôte existent aussi. Un ion de TR interagit avec un autre ion de TR, ou avec un autre ion qui possède une transition résonnante pour un transfert d'énergie. Ces mécanismes d'interaction supplémentaires affectent les durées de vie et les intensités de fluorescence. Un processus d'interaction typique est le transfert d'énergie par upconversion, aussi connu sous le terme Addition de Photons par Transfert d'Energie (APTE). L'APTE décrit le transfert d'énergie entre deux atomes $A$ à $B$. En se désexcitant, l'atome $A$ transfère son énergie à l'atome $B$ qui est alors excité. Ce processus peut se répéter, contribuant à exciter l'atome $B$ dans des niveaux d'énergie supérieurs. La relaxation de l'atome $B$ conduit alors à l'émission d'un photon qui a une énergie plus élevée que celle qui a provoqué son excitation initiale. Pour cette raison, ce processus est appelé upconversion. Cependant, il est peu probable que le transfert d'énergie entre deux ions soit exactement résonant. Le plus souvent, le déficit énergétique est comblé par des phonons; ce mécanisme est appelé transfert d'énergie assisté par phonons.

\section{Luminescence des ions de terres rares insérés dans des nanoparticules}

Dans ce paragraphe, nous nous intéressons au cas où le matériau contenant les ions de terres rares est réduit à l'échelle nanométrique. De telles nanoparticules connaissent un intérêt croissant notamment pour des applications biologiques et leur utilisation spécifique pour la bio-imagerie [15]. Dans le cas des nanoparticules semi-conductrices, la longueur d'onde d'émission est déterminée en modifiant la taille des nanoparticules. Cet effet, dû au confinement quantique, décale les émissions vers les plus courtes longueurs d'onde lorsque la taille des nanoparticules diminue. Une modification de la largeur de la bande interdite a aussi été mise en évidence dans des nanoparticules d'oxydes $\left(\mathrm{Gd}_{2} \mathrm{O}_{3}\right)$ mais reste négligeable [16]. Les modifications des propriétés de luminescence des ions de TR liées à leur incorporation dans des nanoparticules sont principalement liées aux modifications du spectre des phonons, aux changements structuraux ainsi qu'aux interactions avec la surface des particules.

\subsection{Modification du spectre des phonons}


La principale modification attendue pour la luminescence des ions de TR dans un nanocristal est liée au changement de la densité d'états des phonons (phonon density of state, PDOS) qui affecte l'interaction électron-phonon. Le modèle de Debye, qui décrit les phonons dans un cristal massif, n'est pas valable à l'échelle du nanomètre. À partir des travaux de Lamb [17] et Tamura [18], Chen et al. [19] ont calculé la PDOS pour des nanocristaux de $\mathrm{Y}_{2} \mathrm{O}_{2} \mathrm{~S}$ de rayon 5, 10 et $20 \mathrm{~nm}$ (Fig. 2). Ils montrent l'émergence d'un effet de confinement qui se manifeste par : (i) une discrétisation de la PDOS et (ii) l'apparition d'une fréquence de coupure $\left(v_{\min }\right)$ des phonons acoustiques. Cette fréquence de coupure s'exprime par :

$$
v_{\min }=\frac{v_{t}}{2 \pi R} \eta_{\min }
$$

où $v_{t}$ est la vitesse du son pour le mode transverse dans les nanoparticules, $R$ est le rayon de la particule et $\eta_{\min }$ est la fréquence minimale réduite. Pour $\eta_{\min }=2,05$, telle que calculée par les auteurs, l'énergie de coupure des phonons est estimée à 8,4 et $2 \mathrm{~cm}^{-1}$ pour des nanoparticules respectivement de 5,10 et $20 \mathrm{~nm}$ de rayon.
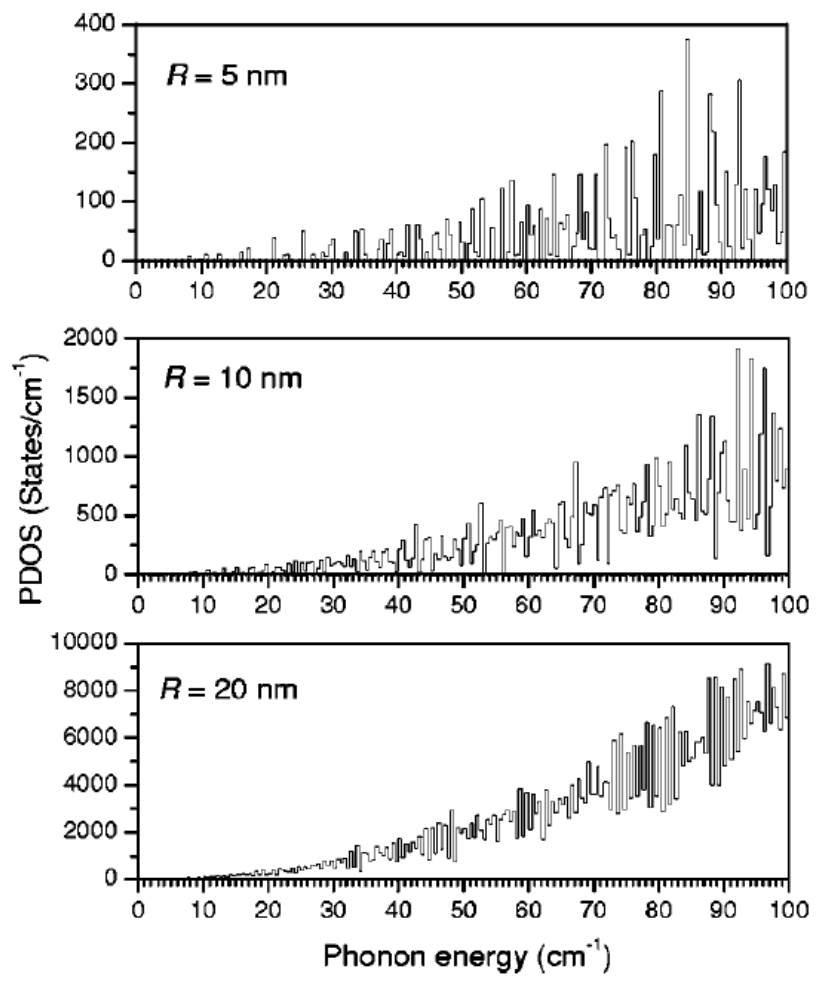

Fig. 2. Densités d'états des phonons calculées pour des nanocristaux de $\mathrm{Y}_{2} \mathrm{O}_{2} \mathrm{~S}$ de rayon $R=5$, 10 et $20 \mathrm{~nm}$ [19]. Reprinted with permission from X. Y. Chen, H. Z. Zhuang, G. K. Liu, S. Li and R. S. Niedbala, Journal of Applied Physics, 94, 5559 (2003). Copyright 2003, American Institute of Physics. [19].

Le changement de PDOS survenant à l'échelle nanométrique est alors attendu pour affecter tous les processus assistés par phonons, tels que la thermalisation Boltzmann des sous-niveaux $4 f$ et les mécanismes de transfert d'énergie assisté par phonons.

La thermalisation des sous-niveaux $4 f$ a été étudiée par Liu et al. dans des nanocristaux de 
$\mathrm{Y}_{2} \mathrm{O}_{2} \mathrm{~S}: \mathrm{Er}^{3+}$ de rayons compris entre 10 et $40 \mathrm{~nm}$ [20]. Dans le cas d'un matériau massif, suite à une excitation vers les niveaux d'énergie supérieure, les électrons relaxent vers le niveau fondamental (le multiplet ${ }^{4} \mathrm{I}_{15 / 2}$ ). La population des différents sous-niveaux (repérés de 1 à 8 sur la Fig. 3) du multiplet ${ }^{4} \mathrm{I}_{15 / 2}$ suit la statistique de Boltzmann par l'intermédiaire du processus d'émission de phonons. A très basse température, $2,6 \mathrm{~K}$, seul le niveau 1 est peuplé comme le montre le spectre d'excitation de la Fig. 3 (la température ne permet pas de peupler les sous-niveaux supérieurs). Pour les nanocristaux à la même température, des bandes d'excitation supplémentaires (« hot bands » de la Fig. 3) sont observées, indiquant que les niveaux 2, 3, 4 et 5 sont aussi peuplés contrairement au matériau massif. Ceci s'explique par le fait que l'énergie de coupure des phonons dans les nanocristaux (énergie estimée à $24 \mathrm{~cm}^{-1}$ dans les nanocristaux de $20 \mathrm{~nm}$ ) ne permet pas à un électron se trouvant sur le niveau 4 par exemple (suite à une désexcitation à partir d'un multiplet d'énergie supérieure) de se relaxer vers les sous-niveaux inférieurs. Ceci est spécifique aux très basses températures. Pour des températures supérieures à $10 \mathrm{~K}$, la population des sous-niveaux pour des nanocristaux est similaire à celle d'un matériau massif, c'est-à-dire répondant à une distribution de Boltzmann.

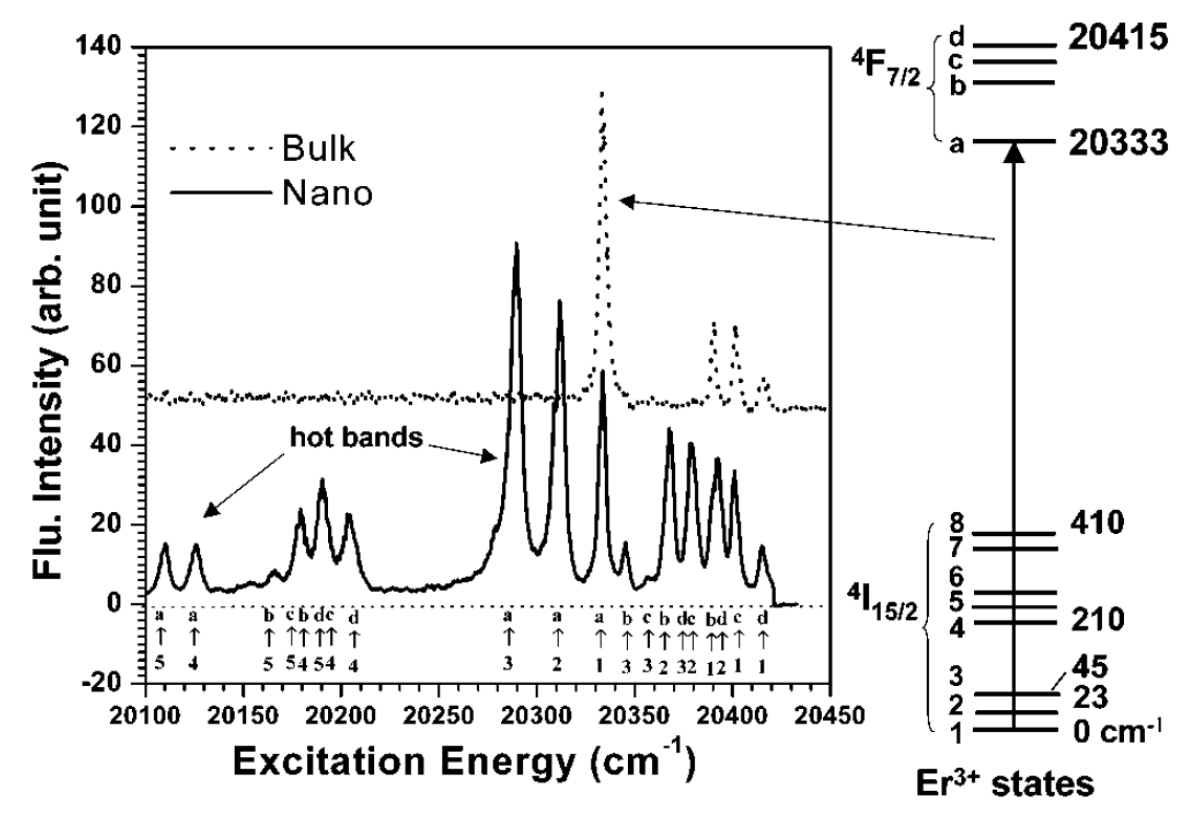

Fig. 3. Spectre d'excitation des ions $\mathrm{Er}^{3+}$ ions dans des nanocristaux de 10-40 $\mathrm{nm}$ (ligne continue) et $400 \mathrm{~nm}$ (ligne pointillée) de diamètre de $\mathrm{Y}_{2} \mathrm{O}_{2} \mathrm{~S}$ à $2,6 \mathrm{~K}$. Le spectre d'excitation des nanoparticules de $400 \mathrm{~nm}$ est assimilé à celui du matériau massif. Les sous-niveaux des deux multiplets ${ }^{4} \mathrm{~F}_{7 / 2}$ et ${ }^{4} \mathrm{I}_{15 / 2}$ des ions $\operatorname{Er}^{3+}$ sont représentés à droite. Le spectre d'excitation est enregistré pour une émission à $18248 \mathrm{~cm}^{-1}$ (issue de l'état excité ${ }^{4} \mathrm{~S}_{3 / 2}$ ) [20]. Reprinted with permission from G.K. Liu, H. Z. Zhuang, and X. Y. Chen, Nano Letters, 2, 535 (2002). Copyright 2002, American Chemical Society.

Les effets sur les transferts d'énergie assistés par phonon ont été étudiés par Chen et al. dans $\mathrm{Y}_{2} \mathrm{O}_{2} \mathrm{~S}$ : $\mathrm{Er}^{3+}$ sous forme massif et de nanocristaux (diamètre de 2 à $30 \mathrm{~nm}$ ) [19]. A température ambiante, ce mécanisme de transfert d'énergie assisté par phonon diminue rapidement avec la diminution de la taille des nanoparticules. Tel que reporté sur la Fig. 4, l'efficacité du transfert d'énergie assisté par 
phonons est de $85 \%$ (par rapport au massif) pour les nanoparticules de $10 \mathrm{~nm}$, diminue à $60 \%$ pour les nanocristaux de $5 \mathrm{~nm}$, et à moins de $10 \%$ pour les nanocristaux de 2,5 nm.

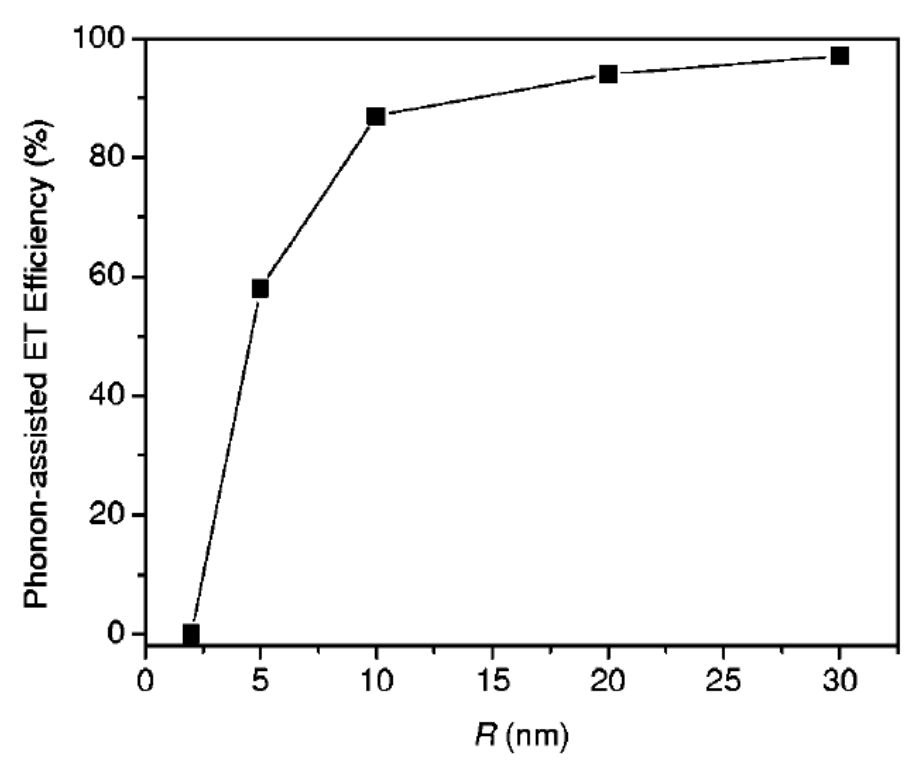

Fig. 4. Efficacité du transfert d'énergie assité par phonons en fonction de la taille des particules dans des nanocristaux de $\mathrm{Y}_{2} \mathrm{O}_{2} \mathrm{~S}: \mathrm{Er}^{3+}$ à $295 \mathrm{~K}$. Pour comparaison, l'efficacité dans un matériau massif est prise à $100 \%$ [19]. Reprinted with permission from X. Y. Chen, H. Z. Zhuang, G. K. Liu, S. Li and R. S. Niedbala, Journal of Applied Physics, 94, 5559 (2003). Copyright 2003, American Institute of Physics.

\subsection{Effets structuraux}

Suivant les conditions de fabrication ou lors de sa croissance, la structure d'une nanoparticule peut évoluer $[21,22,23]$. Les ions de TR sont alors utilisés en tant que sonde structurale pour suivre ces évolutions. Par exemple, dans des nanoparticules de $\mathrm{Gd}_{2} \mathrm{O}_{3}: \mathrm{Eu}^{3+}$, la transition structurale de cubique centrée à monoclinique, lorsque le diamètre dépasse $2,8 \mathrm{~nm}$, est caractérisée par une modification des spectres d'émission des ions $\mathrm{Eu}^{3+}$ [24]. De même, les propriétés de luminescence des ions $\mathrm{Eu}^{3+}$ ont permis de montrer l'influence de la taille et du procédé de préparation sur la structure cristalline des nanoparticules de $\mathrm{Y}_{2} \mathrm{O}_{3}: \mathrm{Eu}^{3+}$ [25]. Dans une autre démarche, l'insertion d'ions de TR a été mise en œuvre pour contrôler la structure cristalline. En effet, le changement de structure cristalline de cubique à hexagonale est obtenue en substituant $\mathrm{Na}^{+}$par $\mathrm{Gd}^{3+}$ dans les nanoparticules de $\mathrm{NaYF}_{4}$ [26].

\subsection{Effets de surface}

Lorsque la taille des particules diminue à l'échelle nanométrique, le rapport surface/volume augmente considérablement. Les interactions entre les ions de TR et la surface (atomes, défauts) des nanoparticules deviennent alors prépondérantes. Un des mécanismes les plus connus est l'extinction de luminescence. Dans le cas de nanocristaux de $\mathrm{NaYF}_{4}$ de $8 \mathrm{~nm}$ de diamètre, il a été montré que les ions de TR distants de moins de 1,7 nm de la surface présentent une extinction de luminescence [27]. De tels effets ont aussi été mis en évidence dans des nanoparticules de YAG: $\mathrm{Ce}^{3+}$, la distance critique étant alors de $7 \mathrm{~nm}$ [28]. Afin de s'affranchir de tels effets néfastes, une solution consiste à enrober la nanoparticule (core) d'une couche vitreuse ou cristalline (shell) pour former une 
nanoparticule dite core-shell [29].

Au-delà des effets d'extinction de luminescence, la présence d'ions de TR en surface présente potentiellement un intérêt car de tels ions peuvent être dans un site de symétrie différente de celle dans le volume. Ainsi, dans une étude menée sur des nanoparticules de $\mathrm{Gd}_{2} \mathrm{O}_{3}: \mathrm{Eu}^{3+}$ [30], les ions europium placés près de la surface émettent à une longueur d'onde différente des ions europium présents dans le volume des nanoparticules.

\section{Influence de la matrice hôte sur la luminescence des nanoparticules dopées d'ions de terres rares}

Après avoir discuté des propriétés optiques des ions de TR dans un matériau massif puis dans des nanoparticules, nous nous intéressons ici au cas des nanoparticules dopées d'ions de TR insérées dans un matériau. Alors que les propriétés de luminescence sont généralement attendues pour être contrôlées par les premiers voisins atomiques, nous verrons que la matrice hôte a une influence sur les propriétés de luminescence des ions de TR bien que ceux-ci en soit éloignés de plusieurs nm.

Lorsque les nanoparticules sont obtenues par traitements thermiques d'un matériau massif, comme dans le cas des vitro-céramiques, la partition des ions de TR dans les nanoparticules est une question primordiale. Les techniques de caractérisations qui ont été mises en œuvre pour le mesurer sont la spectroscopie de fluorescence [31], la diffusion des rayons X (Anomalous Small-Angle Xray Scattering, ASAXS) [32], la spectroscopie électronique (Energy Electron Loss Spectroscopy, EELS) [33], la spectrométrie de masse (SIMS) [34] et plus récemment la Sonde Atomique Tomographique (Atom Probe Tomography, APT) [35].

Les effets présentés dans cette partie sont observés pour des nanocristaux dopés d'ions de TR insérés dans une matrice vitreuse. Dans ces études, la composition chimique des nanocristaux est supposée être indépendante de la taille. Dans le cas des nanoparticules amorphes, la composition peut évoluer en fonction de la taille mais les modifications des propriétés de luminescence des ions de TR dans des nanoparticules amorphes obtenues par traitement thermique sont encore très peu étudiées $[35,36]$.

\subsection{Influence de l'indice de réfraction de la matrice sur la durée de vie radiative}

Comme il a déjà été exprimé dans le paragraphe 2 , la durée de vie radiative des ions de TR dépend de l'indice de réfraction $n$ et donc de la permittivité diélectrique $\varepsilon\left(\varepsilon=n^{2}\right)$ qui sont des grandeurs macroscopiques. Quelle est la pertinence de ces grandeurs à l'échelle nanométrique ? Il a été démontré que la relation de Clausius-Mossotti, qui relie la permittivité diélectrique $\varepsilon$ à la polarisabilité microscopique $\alpha$ des atomes dans un matériau massif :

$$
\frac{\epsilon-1}{\epsilon+2}=\frac{N \alpha}{3}
$$

où $N$ est le nombre de molécules par unité de volume [37], est valide pour des particules aussi petites que 1-2 nm [38]. Une autre question concerne l'étendue spatiale de l'indice de réfraction $n$ et son influence sur l'ion luminescent. Dans leur travail sur un système modèle de nanocouches de $\mathrm{TiO}_{2}$ empilées sur une monocouche de $\mathrm{Gd}_{2} \mathrm{O}_{3}: \mathrm{Eu}^{3+}$ [39], Lebihan et al. ont déduit que, au-delà d'une 
épaisseur proche de $\lambda / 4$ ( $\lambda$ étant la longueur d'onde de la lumière), l'indice de réfraction $n$ de $\mathrm{TiO}_{2}$ n'affecte plus les propriétés optiques d'émission de $\mathrm{Eu}^{3+}$. Ainsi, si l'on considère une émission bleue par exemple, $\lambda=400 \mathrm{~nm}$, l'effet de l'indice de réfraction de l'hôte environnant ne peut pas être négligé pour des particules d'un rayon inférieur à $100 \mathrm{~nm}$.

Dans le cas d'un matériau hétérogène, la durée de vie radiative peut être estimée en considérant un indice effectif $n_{\text {eff }}$ :

$$
\tau_{l o c}=\frac{\tau_{v a c}}{L^{2} n_{e f f}}
$$

où $\tau_{\text {vac }}$ est la durée de vie radiative de l'ion luminescent dans le vide et $L$ est le facteur de correction du champ local.

L'indice de réfraction effectif $n_{\text {eff }}$ et la permittivité diélectrique effective $\varepsilon_{\text {eff }}$ sont définis en utilisant le modèle du milieu effectif. Une variété de modèles permettent de calculer $\varepsilon_{\text {eff }}$ (ou $n_{\text {eff }}$ ) pour des géométries composites spécifiques. En particulier, la géométrie Maxwell-Garnett traite le cas de petites particules incluses dans un matériau hôte, une configuration qui pourrait être assimilée au processus de nucléation/croissance. En géométrie Maxwell-Garnett les nanoparticules sont plus petites que la longueur d'onde optique, et la distance entre chaque particule et son voisin le plus proche doivent être beaucoup plus grande que la taille caractéristique des nanoparticules. Dans ces conditions, $\varepsilon_{\text {eff }}$ est exprimée comme:

$$
\frac{\epsilon_{e f f}-\epsilon_{h}}{\epsilon_{e f f}+2 \epsilon_{h}}=f_{n p} \frac{\epsilon_{n p}-\epsilon_{h}}{\epsilon_{n p}+2 \epsilon_{h}}
$$

où $\varepsilon_{h}$ et $\varepsilon_{n p}$ sont les constantes diélectriques de l'hôte et de la nanoparticule, et $f_{n p}$ est la fraction volumique de nanoparticules, et doit être $<<1$.

En géométrie Bruggeman, le matériau est considéré comme deux phases interconnectées (cas de la décomposition spinodale) avec une phase contenant l'ion de TR. La permittivité diélectrique effective $\varepsilon_{\text {eff }}$ de ce matériau composite est telle que:

$$
f_{1} \frac{\epsilon_{1}-\epsilon_{\text {eff }}}{\epsilon_{1}+2 \epsilon_{\text {eff }}}+f_{2} \frac{\epsilon_{2}-\epsilon_{\text {eff }}}{\epsilon_{2}+2 \epsilon_{\text {eff }}}=0
$$

où $\varepsilon_{1}$ et $\varepsilon_{2}$ sont les constantes diélectriques, $f_{1}$ et $f_{2}$ sont les fractions volumiques des composants 1 et 2 .

Une autre formule utilisée pour estimer l'indice de réfraction effectif est [40]:

$$
n_{\text {eff }}=f_{n p} n_{n p}+\left(1-f_{n p}\right) n_{h}
$$

où $n_{n p}$ et $n_{h}$ sont les indices de réfraction de la nanoparticule et de la matrice hôte.

Pour illustrer l'effet de l'indice de réfraction de la matrice sur les propriétés de luminescence, la variation de la durée de vie radiative de l'ion $\mathrm{Eu}^{3+}$ en fonction de $n$ est rapportée dans Fig. 5 [40]. Dans cette étude, les auteurs ont préparé des nanocristaux de $\mathrm{Y}_{2} \mathrm{O}_{3}: \mathrm{Eu}^{3+} \mathrm{de} \sim 7 \mathrm{~nm}$, immergés dans des liquides de différents indices de réfraction. La durée de vie radiative de fluorescence a été 
mesurée pour deux sites cristallographiques indiqués $\mathrm{A}$ et $\mathrm{C}$ sur la Fig. 5 . Il ressort clairement que la durée de vie radiative augmente avec la diminution de l'indice de réfraction de l'hôte (un liquide dans ce cas).

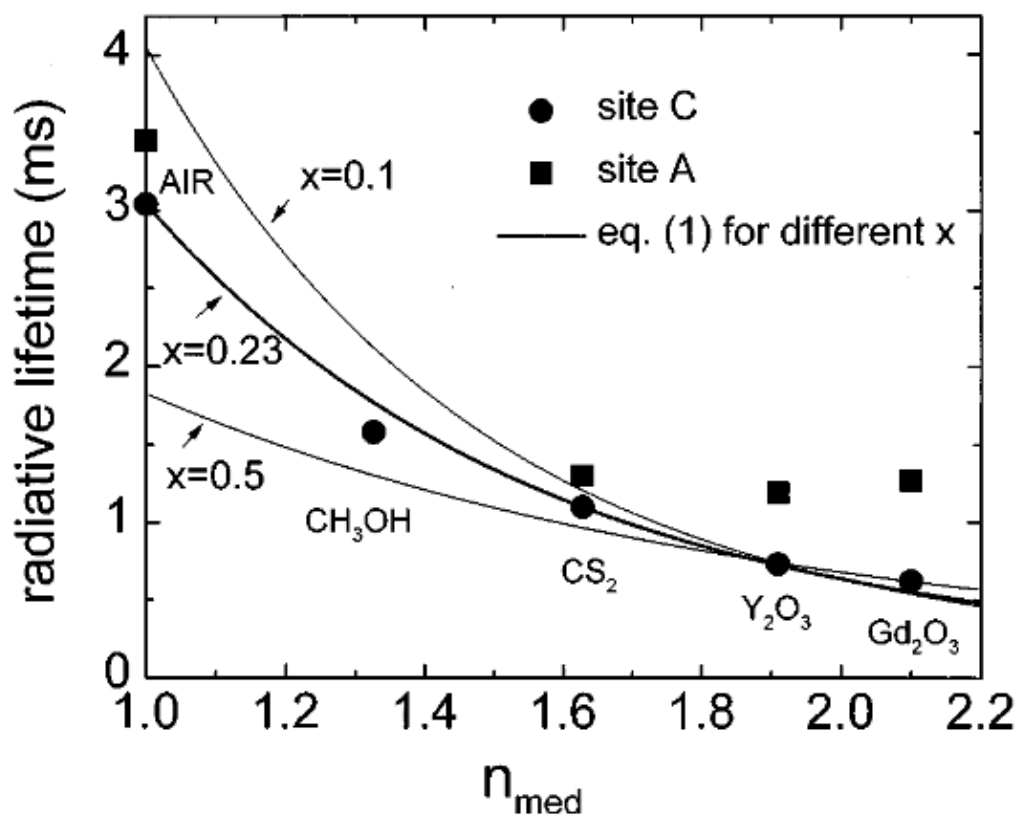

Fig. 5. Variation de la durée de vie radiative du multiplet ${ }^{5} \mathrm{D}_{0}$ de l'ion $\mathrm{Eu}^{3+}$ dans un nanocristal de $\mathrm{Y}_{2} \mathrm{O}_{3}$ (A et $\mathrm{C}$ correspondent à deux sites cristallographiques) suivant l'indice de réfraction du milieu hôte $\left(n_{\text {med }}\right)$ à $\mathrm{T}=295$ $\mathrm{K}$ [40]. Les durées de vie radiatives reportées pour $\mathrm{Y}_{2} \mathrm{O}_{3}$ et $\mathrm{Gd}_{2} \mathrm{O}_{3}$ sont mesurées dans des monocristaux. La ligne continue représente l'ajustement pour les nanocristaux $\left(\mathrm{Eu}^{3+}\right.$ en site $\left.\mathrm{C}\right)$ en considérant $\mathrm{n}_{e f f}(x)=$ $x \times \mathrm{n}\left(\mathrm{Y}_{2} \mathrm{O}_{3}\right)-(1-x) \times \mathrm{n}_{\text {med }}$ avec différentes valeurs de $x$. Reprinted figure with permission from R.S. Meltzer, S.P. Feofilov, B. Tissue, H.B. Yuan, Physical Review B, 60, R14012, 1999. Copyright (1999) by the American Physical Society.

\subsection{Système à deux niveaux}

Lorsque des nanocristaux de $\mathrm{Y}_{2} \mathrm{O}_{3}: \mathrm{Eu}^{3+}$ sont incorporés dans un polymère, ce dernier impacte l'élargissement homogène des niveaux atomiques de $\mathrm{Eu}^{3+}$ (Fig. 6) [41]. La largeur de raie homogène ( $\gamma_{\text {homogeneous }}$ ) a été sondée en utilisant une technique appelée Spectral Hole Burning, la largeur de raie $\gamma_{H B}$ mesurée par cette technique étant reliée à $\gamma_{\text {homogeneous }}$ par: $\gamma_{\text {homogeneous }}=\gamma_{H B} / 2$. Lorsque les nanoparticules sont incorporées dans le polymère, la largeur de raie $\gamma_{H B}$ est plus grande, et la variation est linéaire avec la température $T$, par opposition à la dépendance en $T^{3}$ montrée dans le cas d'un nanocristal isolé (non inséré dans une matrice). Une observation semblable à celle du polymère a été faite dans un verre [2]. 


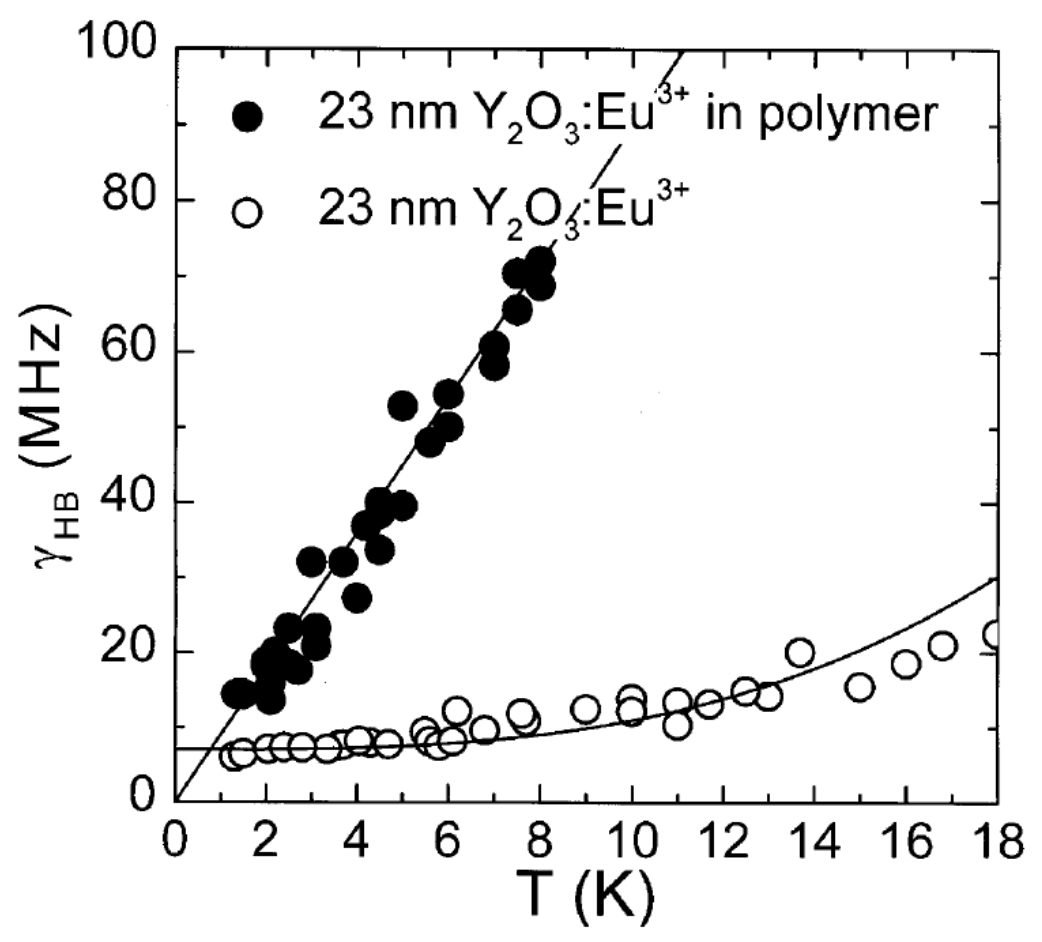

Fig. 6. Variation en fonction de la température de la largeur de raie $\gamma_{H B}$ pour les ions $\mathrm{Eu}^{3+}$ dopés dans des nanocristaux de $\mathrm{Y}_{2} \mathrm{O}_{3}$. Ligne continue : $\gamma_{H B}$ varie comme $\sim T$ et $\sim T^{3}$ quand les nanoparticules dopées d'ions de TR sont respectivement insérées dans un polymère ou isolées. [41]. Reprinted figure with permission from R.S. Meltzer, W.M. Yen, H. Zheng, S.P. Feofilov, M.J. Dejneka, B.M. Tissue, H.B. Yuan, Physical Review B, 64, 100201, 2001. Copyright (2001) by the American Physical Society.

Les résultats présentés dans la Fig. 6 ont été interprétés à l'aide du modèle à deux niveaux (TwoLevel Systems, TLS) [42]. Ce modèle a été initialement développé pour expliquer la chaleur spécifique à basse température et la conductivité thermique du verre [43]. Contrairement au cristal parfait (les atomes occupent une position unique), les atomes du verre (ou d'un système désordonné) se déplacent dans un potentiel ayant plusieurs minima. Ils peuvent passer d'un minimum à un autre par effet tunnel. Ces différentes positions influent sur l'interaction entre les ions de TR et les atomes de la matrice hôte. En considérant ce modèle, l'élargissement homogène est calculé en supposant une interaction dipôle-dipôle entre les ions de TR et les niveaux TLS des atomes de la matrice hôte. De légères modifications dans l'éclatement des niveaux d'énergie des TLS provoquent l'élargissement de la largeur de raie homogène. L'accord avec les données expérimentales mesurées par R.S. Meltzer et al. est particulièrement important lorsque les nanocristaux sont supposés être sans TLS, à savoir, l'élargissement homogène est due uniquement à l'interaction avec les TLS situés à l'extérieur du nanocristal contenant les ions de TR. Cela révèle que l'interaction TLS est à longue portée. 


\subsection{Interaction avec les "phonons" de la matrice vitreuses environnante}

L'apparition d'une fréquence de coupure pour les nanoparticules a été discutée dans le paragraphe 3.1. Il a été montré que cet effet diminue le taux de relaxation à un phonon entre sous-niveaux Stark rapprochés. Cet effet est contesté dans une étude réalisée par Meltzer et al. sur des nanocristaux de $\mathrm{LaF}_{3}: \mathrm{Ho}^{3+}$ de différentes tailles présents dans une matrice de silicate [44]. Le but de leur étude était de mesurer le temps de relaxation entre les deux sous-niveaux les plus bas ${ }^{5} \mathrm{~F}_{5}$, espacés de $14,5 \mathrm{~cm}^{-1}$ (Fig. 7a). Les auteurs ont montré que le temps de relaxation augmente avec une augmentation de la taille des particules. Lorsque la taille de particule atteint $25 \mathrm{~nm}$, le temps de relaxation est proche de celui attendu pour le monocristal de $\mathrm{LaF}_{3}$ (Fig. 7b). L'explication des auteurs pour ces résultats inattendus est qu'il existe une interaction entre les états électroniques des ions de TR présents dans les nanocristaux et les modes de vibration de la matrice vitreuse. Cela implique que les modes de vibration de la matrice vitreuse et les phonons des nanocristaux peuvent former de nouveaux états de vibration mixtes qui interagissent avec les ions de TR. Ce type d'interaction a également été montré pour impacter les mécanismes d'upconversion dans des nanocristaux de $\mathrm{LaF}_{3}: \mathrm{Er}^{3+}$ au sein d'une vitro-céramique d'oxyfluorure [45].

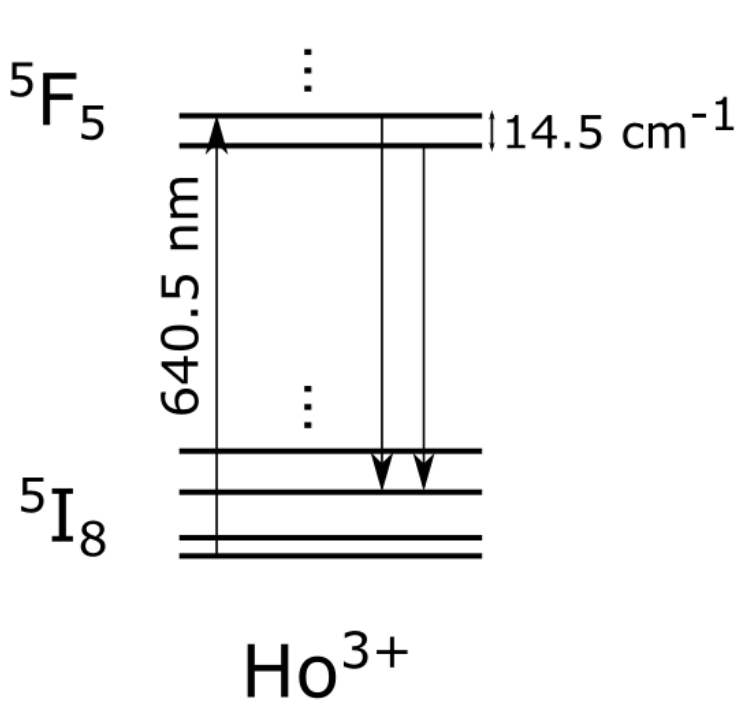

(a)

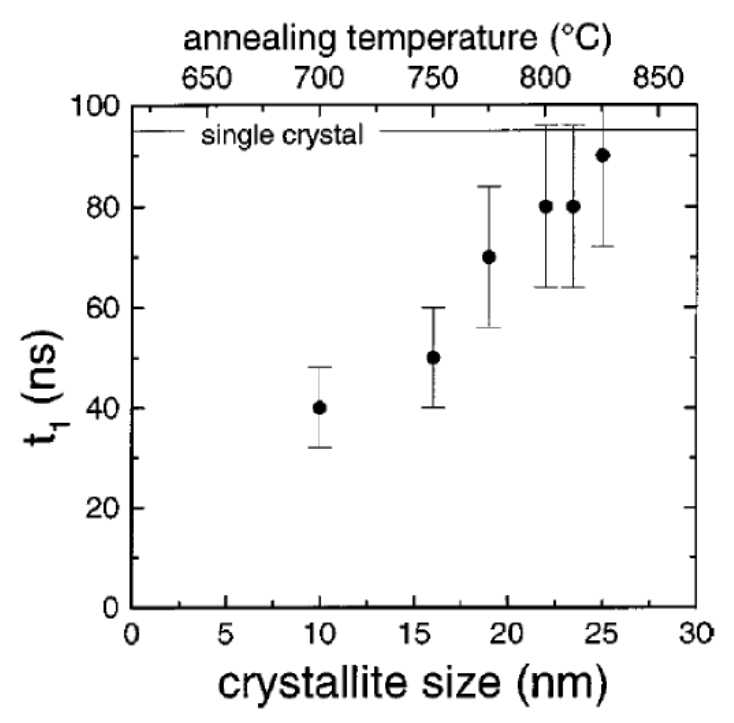

(b)

Fig. 7. Nanocristaux dopés $\mathrm{Ho}^{3+}$ de différentes tailles, présents dans une vitro-céramique d'oxy-fluorure obtenue par recuit à différentes températures. (a) Diagramme des niveaux d'énergie de l'ion $\mathrm{Ho}^{3+}$, seuls les deux premiers multiplets $\left({ }^{5} \mathrm{I}_{8}\right.$ et $\left.{ }^{5} \mathrm{~F}_{5}\right)$ sont représentés. (b) Temps de relaxation $t_{l}$ entre les deux plus bas sousniveaux de ${ }^{5} \mathrm{~F}_{5}$ de $\mathrm{Ho}^{3+}$ (séparés par 14,5 $\mathrm{cm}^{-1}$ ). Ligne continue: valeur du temps de relaxation pour un monocristal de $\mathrm{LaF}_{3}$ [44]. Les auteurs ont excité le second plus bas sous-niveau de ${ }^{5} \mathrm{~F}_{5}$ de $\mathrm{Ho}^{3+}(\lambda=640,5 \mathrm{~nm})$ et mesuré l'intensité de la transition vers les deux plus bas sous-niveaux de ${ }^{5} \mathrm{I}_{8}$ (séparés par $14,5 \mathrm{~cm}^{-1}$ ). Reprinted figure with permission from R.S. Meltzer, W.M. Yen, H. Zheng, S.P. Feofilov, M.J. Dejneka, Physical Review B, 66, 224202, 2002. Copyright (2002) by the American Physical Society. 


\section{Conclusion}

Les propriétés de luminescence uniques des ions de TR permettent le développement de matériaux optiques avec un large éventail d'applications telles que l'éclairage, les télécommunications, le stockage optique, la bio-imagerie, etc. L'optimisation des propriétés de luminescence est principalement basée sur les interactions entre les ions de TR et leur environnement. Les propriétés spectroscopiques des ions de TR dans des matériaux massifs ont été abondamment étudiées jusqu'à maintenant. Avec l'introduction des nanotechnologies, les propriétés spectroscopiques des ions de TR ont gagné un regain d'intérêt. De nombreuses études scientifiques récentes ont porté sur les propriétés spectroscopiques des ions de TR dans des nanoparticules, et comment elles diffèrent des propriétés spectroscopiques des ions de TR dans des matériaux massifs. Les principales différences sont dues à des changements dans la densité d'états des phonons et des effets de surface. Il a aussi été montré que les mécanismes impliqués dans des nanoparticules dopées d'ions de TR isolées ou incorporées dans un matériau sont différents. Des paramètres tels que l'indice de réfraction, les états de vibration et les états à deux niveaux de la matrice hôte interférent avec les ions de TR et modifient ses propriétés de luminescence.

La compréhension de ces différents phénomènes permet d'ouvrir de nouvelles perspectives pour la photonique. La mise en application passera par un contrôle et une optimisation des procédés de fabrication. De plus, dans le cas des vitro-céramiques, le contrôle de la diffusion de la lumière sera primordiale pour profiter pleinement des améliorations apportées par les nanoparticules.

\section{Références bibliographiques}


[1] Chakhmouradian A.R., Walls F. (2012) "Rare earth elements: minerals, mines, magnets (and more)." Elements, O, 333-340.

[2] Liu G., Jacquier B. (2005) "Spectroscopic properties of rare earths in optical materials." Springer Series in Materials Science. Springer.

[3] Dwivedi Y., Zilio S.C. (2014) "Advances in rare earth spectroscopy and applications." J. Nanosci. Nanotechnol., 14, 1578-1596.

[4] Digonnet M.J.F. (2001) “Rare-earth-doped fiber lasers and amplifiers.” CRC Press.

[5] Auzel, F., Pecile, D., Morin, D. (1975) « Rare Earth Doped Vitroceramics: New, Efficient, Blue and Green Emitting Materials for Infrared Up-Conversion.» J. Electrochem. Soc., 122, 101-107.

[6] Wang, Y., Ohwaki, J. (1993) « New transparent vitroceramics codoped with $\mathrm{Er}^{3+}$ and $\mathrm{Yb}^{3+}$ for efficient frequency upconversion.» Appl. Phys. Lett., 63, 3268-3270.

[7] Gredin P., Mortier M. (2013) «Vitrocéramiques oxyfluorées » in «Du verre au cristal, nucléation, croissance et démixtion, de la recherche aux applications. », Ed. D.R. Neuville, L. Cormier, D. Caurant, L. Montagne, EDP Sciences.

[8] Goncalves A.C., Santos L.F., Almeida R.M. (2002) "Rare-earth-doped transparent glass ceramics." C. R. Chim., 5, 845-854.

[9] Boulard B., Van T.T.T., Łukowiak A., Bouajaj A., Rocha Gonçalves R., Chiappini A., Chiasera A., Blanc W., Duran A., Turrell S., Prudenzano F., Scotognella F., Ramponi R., Marciniak M., Righini G.C., Ferrari M. (2015) "Photonic glass-ceramics: consolidated outcomes and prospects", Proc SPIE Oxide-based Materials and Devices VI, edited by Ferechteh H. Teherani, David C. Look, David J. Rogers (Photonic West) Vol. 9364, 93640Z-01-93640Z-10

[10] Komatsu, T., Honma, T. (2013) « Optical Active Nano-Glass-Ceramics.». Int. J. Appl. Glass Sci., 4, 125-135.

[11] Rogers E., Dorenbos P., Van der Kolk E. (2011) "Systematics in the optical and electronic properties of the binary lanthanide halide, chalcogenide and pnictide compounds : an overview." New J. Phys., 13, 093038.

[12] Dorenbos P. (2013) "Lanthanide 4f-electron binding energies and the nephelauxetic effect in wide band gap compounds.” J. Lumin., 136, 122-129.

[13] Dodson C.M., Zia R. (2012) "Magnetic dipole and electric quadrupole transitions in the trivalent lanthanide series: Calculated emission rates and oscillator strengths.” Phys. Rev. B, 86, 125102.

[14] Schmidt T., Macfarlane R.M., Völker S. (1994) "Persistent and transient spectral hole burning in $\mathrm{Pr}^{3+}$-and $\mathrm{Eu}^{3+}$-doped silicate glasses.” Phys. Rev. B, 50, 15707-15718.

[15] Li X., Zhang F., Zhao D. (2013) "Highly efficient lanthanide upconverting nanomaterials : Progresses and challenges.” Nano Today, 8, 643-676.

[16] Mercier B., Ledoux G., Dujardin C., Nicolas D., Masenelli B., Melinon P., Bergeret G. (2007) "Quantum confinement effect on $\mathrm{Gd}_{2} \mathrm{O}_{3}$ clusters.” J. Chem. Phys., 126, 044507.

[17] Lamb H. (1882) "On the vibrations of an elastic sphere." Proc. Math. Soc. London, 13, 189212. 
[18] Tamura A. (1995) "Smoothed density of states of electrons and smoothed frequency spectrum of phonons for a mesoscopic system.” Phys. Rev. B, 52, 2668-2676.

[19] Chen X.Y., Zhuang H.Z., Liu G.K., Li S., Niedbala R.S. (2003) “Confinement on energy transfer between luminescent centers in nanocrystals.” J. Appl. Phys., 84, 5559-5565.

[20] Liu G.K., Zhuang H.Z., Chen X.Y. (2003) "Restricted phonon relaxation and anomalous thermalization of rare earth ions in nanocrystals." Nanolett., 2, 535-539.

[21] Ray S., León-Luis S.F., Manjón F.J., Mollar M.A., Gomis Ó., Rodríguez-Mendoza U.R., Lavín V. (2014) "Broadband, site selective and time resolved photoluminescence spectroscopic studies of finely size-modulated $\mathrm{Y}_{2} \mathrm{O}_{3}: \mathrm{Eu}^{3+}$ phosphors synthesized by a complex based precursor solution method." Curr. Appl. Phys., 14, 72-81.

[22] Dey A., Bomans P.H., Müller F.A., Will J., Frederik P.M., de With G., Sommerdijk N.A. (2010) "The role of prenucleation clusters in surface-induced calcium phosphate crystallization." Nat. Mater., 9, 1010-1014.

[23] Chung S.Y., Kim Y.M., Kim J.G., Kim Y.J. (2009) "Multiphase transformation and Ostwald's rule of stages during crystallization of a metal phosphate." Nature Phys., 5, 68-73.

[24] Nicolas D., Masenelli B., Melinon P., Bernstein E., Dujardin C., Ledoux G., Esnouf C. (2006) "Structural transition in rare earth oxide clusters." J. Chem. Phys., 125, 171104.

[25] Tissue B.M., Yuan H.B. (2003) "Structure, particle size, and annealing of gas phase-condensed $\mathrm{Eu}^{3+}: \mathrm{Y}_{2} \mathrm{O}_{3}$ nanophosphors.” J. Solid St. Chem., 171, 12-18.

[26] Wang F., Han Y., Lim C.S., Lu Y., Wang J., Xu J., Chen H., Zhang C., Hong M., Liu X. (2010) "Simultaneous phase and size control of upconversion nanocrystals through lanthanide doping." Nature, 463, 1061-1065.

[27] Gargas D.J., Chan E.M., Ostrowski A.D., Aloni S., Altoe M.V.P., Barnard E.S., Sanii B., Urban J.J., Milliron D.J., Cohen B.E., Schuck P.J. (2014) "Engineering bright sub-10-nm upconverting nanocrystals for single-molecule imaging.” Nat. Nano., 9, 300-305.

[28] Tian L.J., Sun Y.J., Yu Y., Kong X.G., Zhang H. (2008) "Surface effect of nano-phosphors studied by time-resolved spectroscopy of $\mathrm{Ce}^{3+}$." Chem. Phys. Lett., 452, 188-192.

[29] Sudarsan V., Van Veggel F.C., Herring R.A., Raudsepp M. (2005) "Surface $\mathrm{Eu}^{3+}$ ions are different than "bulk" $\mathrm{Eu}^{3+}$ ions in crystalline doped $\mathrm{LaF}_{3}$ nanoparticles." J. Mater. Chem., 15, 13321342 .

[30] Ledoux G., Mercier B., Louis C., Dujardin C., Tillement O., Perriat P. (2004) "Synthesis and optical characterization of $\mathrm{Gd}_{2} \mathrm{O}_{3}: \mathrm{Eu}^{3+}$ nanocrystals: surface states and VUV excitation." Radiat. Meas., 38, 763-766.

[31] Jones G.C., Houde-Walter S.N. (2005) "Erbium partitioning in a heavily doped transparent glass ceramic.” Opt. Lett., 30, 2122-2124.

[32] Haas S., Hoell A., Wurth R., Rüssel C., Boesecke P., Vainio U. (2010) "Analysis of nanostructure and nanochemistry by ASAXS : accessing phase composition of oxyfluoride glass ceramics doped with $\mathrm{Er}^{3+} / \mathrm{Yb}^{3+}$.” Phys. Rev. B, 81, 184207.

[33] Liu C., Zhao X., Heo J. (2013) "Direct imaging of inhomogeneous distribution of $\mathrm{Er}^{3+}$ ions in lead fluoride nanocrystals.” J. Non-Cryst. Solids, 365, 1-5. 
[34] Blanc W., Guillermier C., Dussardier B. (2012) "Composition of nanoparticles in optical fibers by secondary ion mass spectrometry.” Opt. Mater. Express, 2, 1504-1510.

[35] Francois-Saint-Cyr H., Martin I., Blanc W., LeCoustumer P., Hombourger C., Neuville D., Larson D. J., Prosa T. J. and Guillermier C. (2014) "Correlative Compositional Analysis of FiberOptic Nanoparticles.” Microsc. Microanal., 20, 994-995.

[36] D'Acapito F., Blanc W., Dussardier B. (2014) "Different Er ${ }^{3+}$ environments in Mg-based nanoparticle-doped optical fibre preforms.” J. Non-Cryst. Solids Soc., 401, 50-53

[37] Born M. and Wolf E. (1999) “Principles of optics.” Cambridge University Press.

[38] Aspnes D.E. (1982) "Bounds to average internal fields in two-component composites." Phys. Rev. Lett., 48, 1629-1632.

[39] LeBihan V., Pillonnet A., Amans D., Ledoux G., Marty O., Dujardin C. (2008) "Critical dimension where the macroscopic definition of refractive index can be applied at a nanometric scale." Phys. Rev. B, 78, 113405.

[40] Meltzer R.S., Feofilov S.P., Tissue B., Yuan H.B. (1999) "Dependence of fluorescence lifetimes of $\mathrm{Y}_{2} \mathrm{O}_{3}: \mathrm{Eu}^{3+}$ nanoparticles on the surrounding medium." Phys. Rev. B, 60, R14012R14015.

[41] Meltzer R.S., Yen W.M., Zheng H., Feofilov S.P., Dejneka M.J., Tissue B.M., Yuan H.B. (2001) "Evidence for long-range interactions between rare-earth impurity ions in nanocrystals embedded in amorphous matrices with the two-level systems of the matrix." Phys. Rev. B, 64, 100201-10020.

[42] Lyo S.K. (1982) “Anomalous optical homogeneous linewidths in glasses.” Phys. Rev. Lett., 48, 688-691.

[43] Philips W.A. (1987) “Two-level states in glasses.” Rep. Prog. Phys., 50, 1657-1708.

[44] Meltzer R.S., Yen W.M., Zheng H., Feofilov S.P., Dejneka M.J. (2002) "Relaxation between closely spaced electronic levels of rare-earth ions doped in nanocrystals embedded in glass." Phys. Rev. B, 66, 224202.

[45] Ma E., Hu Z., Wang Y., Bao F. (2006) "Influence of structural evolution on fluorescence properties of transparent glass ceramics containing $\mathrm{LaF}_{3}$ nanocrystals.” J. Lumin., 118, 131-138. 